\title{
The Benefits of Combination Therapy with Esomeprazole and Rebamipide in Symptom Improvement in Reflux Esophagitis: An International Multicenter Study
}

Su Jin Hong ${ }^{1}$, Soo-Heon Park², Jeong Seop Moon ${ }^{3}$, Woon Geon Shin ${ }^{4}$, Jae Gyu Kim ${ }^{5}$, Yong Chan Lee ${ }^{6}$, Dong Ho Lee ${ }^{7}$, Jae Young Jang ${ }^{8}$, Jae J. Kim ${ }^{9}$, Hang-Lak Lee ${ }^{10}$, Sang Woo Lee ${ }^{11}$, Young Hwangbo ${ }^{12}$, Jianming Xu ${ }^{13}$, Bangmao Wang ${ }^{14}$, Zhanxiong Xue ${ }^{15}$, Fei Liu ${ }^{16}$, Yaozong Yuan ${ }^{17}$, Somchai Leelakusolvong ${ }^{18}$, and Frederick Dy ${ }^{19}$

${ }^{1}$ Department of Internal Medicine, Soonchunhyang University Bucheon Hospital, Soonchunhyang University College of Medicine, Bucheon, ${ }^{2}$ Department of Internal Medicine, The Catholic University of Korea College of Medicine, ${ }^{3}$ Department of Internal Medicine, Inje University Seoul Paik Hospital, Inje University College of Medicine, ${ }^{4}$ Department of Internal Medicine, Hallym University College of Medicine, ${ }^{5}$ Department of Internal Medicine, Chung-Ang University College of Medicine, ${ }^{6}$ Department of Internal Medicine, Yonsei University College of Medicine, Seoul, ${ }^{7}$ Department of Internal Medicine, Seoul National University Bundang Hospital, Seongnam, ${ }^{8}$ Department of Internal Medicine, Kyung Hee University School of Medicine, ${ }^{9}$ Department of Medicine, Samsung Medical Center, Sungkyunkwan University School of Medicine, ${ }^{10}$ Department of Internal Medicine, Hanyang University College of Medicine, Seoul, ${ }^{11}$ Department of Internal Medicine, Korea University Ansan Hospital, Korea University College of Medicine, Ansan, ${ }^{12}$ Department of Preventive Medicine, Soonchunhyang University College of Medicine, Cheonan, Korea, ${ }^{13}$ Department of Gastroenterology, The First Affiliated Hospital, Anhui Medical University, Hefei, ${ }^{14}$ Department of Gastroenterology and Hepatology, General Hospital, Tianjin Medical University, Tianjin, ${ }^{15}$ Department of Gastroenterology, The Second Affiliated Hospital and Yuying Children's Hospital, Wenzhou Medical University, Wenzhou, ${ }^{16}$ Department of Gastroenterology, Shanghai East Hospital, Tongji University, ${ }^{17}$ Department of Gastroenterology, Ruijin Hospital, Shanghai Jiaotong University, Shanghi, China, ${ }^{18}$ Department of Internal Medicine, Siriraj Hospital, Mahidol University, Bangkok, Thailand, and ${ }^{19}$ Department of Internal Medicine, University of Santo Tomas Hospital, Manila, Philippines

Background/Aims: To investigate the effects of esomeprazole and rebamipide combination therapy on symptomatic improvement in patients with reflux esophagitis. Methods: A total of 501 patients with reflux esophagitis were randomized into one of the following two treatment regimens: $40 \mathrm{mg}$ esomeprazole plus $300 \mathrm{mg}$ rebamipide daily (combination therapy group) or $40 \mathrm{mg}$ esomeprazole daily (monotherapy group). We used a symptom questionnaire that evaluated heartburn, acid regurgitation, and four upper gastrointestinal symptoms. The primary efficacy end point was the mean decrease in the total symptom score. Results: The mean decreases in the total symptom score at 4 weeks were estimated to be $-18.1 \pm 13.8$ in the combination therapy group and $-15.1 \pm 11.9$ in the monotherapy group $(p=0.011)$. Changes in reflux symptoms from baseline after 4 weeks of treatment were $-8.4 \pm 6.6$ in the combination therapy group and $-6.8 \pm 5.9$ in the monotherapy group $(p=0.009)$. Conclusions: Over a 4-week treatment course, esomeprazole and rebamipide combination therapy was more effective in decreasing the symptoms of reflux esophagitis than esomeprazole monotherapy. (Gut Liver 2016;10:910-916)
Key Words: Peptic esophagitis; Symptoms; Heartburn; Rebamipide; Esomeprazole

\section{INTRODUCTION}

Gastroesophageal reflux disease (GERD) involves gastroesophageal reflux of gastroduodenal contents, including gastric acid, bile, pepsin, and duodenal contents, which provokes tissue damage and inflammation in the normal mucous membranes of the esophagus, manifesting with clinical symptoms. ${ }^{1}$ Reflux esophagitis is characterized by esophageal erosions and can result in complications, such as esophageal stricture, Barrett's esophagus, and esophageal adenocarcinoma. GERD has been associated with infiltration of inflammatory cells and expression of interleukin (IL)-8 in esophageal mucosa. ${ }^{2}$

Proton pump inhibitors (PPIs) are used widely as an optimal treatment to treat reflux esophagitis. In addition, there is a medication that can reduce the inflammatory process effectively and improve symptoms of reflux esophagitis. The healing rate of erosive esophagitis was demonstrated $81 \%$ and $96 \%$ at 4 and

\footnotetext{
Correspondence to: Soo-Heon Park

Department of Internal Medicine, Yeouido St. Mary's Hospital, The Catholic University of Korea College of Medicine, 10 63-ro, Yeongdeungpo-gu, Seoul 07345, Korea

Tel: +82-2-3779-1163, Fax: +82-2-3779-1331, E-mail: psheon5132@naver.com

Received on October 22, 2015. Revised on January 2, 2016. Accepted on January 2, 2016. Published online June 13, 2016

pISSN 1976-2283 eISSN 2005-1212 https://doi.org/10.5009/gnl15537

@. This is an Open Access article distributed under the terms of the Creative Commons Attribution Non-Commercial License (http://creativecommons.org/licenses/by-nc/4.0) which permits unrestricted non-commercial use, distribution, and reproduction in any medium, provided the original work is properly cited.
} 
8 weeks in the patients receiving esomeprazole $40 \mathrm{mg}$, respectively. ${ }^{3}$ Meanwhile, relief of symptoms was reported as a predictor of healing of erosive esophagitis in previous study. ${ }^{4}$ Some patients with GERD fail to recover from erosive esophagitis or have continuing reflux symptoms, despite the use of PPIs. Thus, a new kind of drug is needed to treat patients with GERD.

Rebamipide, a drug that stimulates the synthesis of prostaglandin and mucous glycoprotein, inhibits active oxygen, inflammatory cytokines and chemokines, and suppresses the activity of leukocytes, is recognized as a representative defensive medication against inflammatory processes. ${ }^{5}$ In addition, a portion of reflux esophagitis patients have gastritis; ${ }^{6}$ rebamipide, as an effective therapeutic agent for gastritis, led to effective symptomatic responses and improvement of endoscopic and histologic parameters in chronic gastritis patients with dyspepsia refractory to PPIs. ${ }^{7}$ Rebamipide is a candidate drug for the reduction of inflammation in the esophageal mucosa of patients with GERD. Decreasing inflammation is believed to help maintain mucosal resistance of the esophagus. One study showed that IL-8 messenger RNA (mRNA) expression decreased significantly in the esophageal mucosa of patients taking rebamipide, compared with that of patients not taking rebamipide. ${ }^{8}$

We hypothesized that rebamipide would help alleviate symptoms in patients with GERD. Because erosive esophagitis can exclude functional heartburn without the need for an ambulatory $\mathrm{pH}$-impedance study, we included patients with erosive esophagitis in this study. And, we planned to use symptom relief as an indicator which estimates the treatment response in our study.

\section{MATERIALS AND METHODS}

\section{Patients}

This randomized, prospective, multicenter, and open-label trial was conducted in 18 hospitals in Korea, China, Thailand, and the Philippines. Patients with a diagnosis of reflux esophagitis were enrolled consecutively between November 2011 and April 2014. The study protocol was approved by the Institutional Review Board of each hospital and was registered with the University Hospital Medical Information Network (UMIN) Clinical Trial Registry as UMIN000005602.

Patients between the ages of 20 and 69 years were investigated for the presence of reflux symptoms. Endoscopy was performed only in patients with heartburn or acid regurgitation exceeding a frequency of two episodes per week. Erosive reflux esophagitis was diagnosed and classified according to the Los Angeles classification. ${ }^{9}$ Endoscopic gastritis was evaluated by the endoscopic division of the Sydney System. ${ }^{10}$ For Helicobacter pylori infection testing, the $H$. pylori status was defined if the urease test and histopathologic results were both negative or positive for infection. Representative endoscopic images were obtained and stored as digital files. The review of endoscopic pictures to determine eligible patients for enrollment was conducted by a single investigator (S.J.H.) to control for interobserver variability in the interpretation of reflux esophagitis and gastritis.

Patients were excluded if they were pregnant or lactating, were allergic to esomeprazole or rebamipide, had a serious comorbidity (e.g., cardiovascular disease, lung disease, hematological disease, endocrine disease, liver cirrhosis, end-stage renal disease, major psychiatric disease, or alcohol or drug addiction), were unwilling to participate, or had received PPIs, low-dose aspirin, or nonsteroidal anti-inflammatory drugs in the previous 1 month. The body mass index (BMI), smoking status, and alcohol use of each patient at baseline were recorded. All participants provided written informed consent.

\section{Randomization and compliance}

Random allocation lists (1:1 ratio) were generated by a computer program and distributed to each institution. Based on the tables, the subjects were randomly allocated to the experimental and control groups. According to the allocation, patient numbers were assigned and used by the investigators to describe the investigational products provided to the subjects by clinical trial pharmacists. At baseline, a sufficient supply of study medication was administered. The patients in the experimental group (Combination therapy group) received 40-mg esomeprazole strontium (Esomezol; Hanmi Pharmaceutical Co., Ltd., Seoul, Korea) and 300-mg rebamipide (Mucosta ${ }^{\circledR}$; Korea Otsuka Pharmaceutical Co., Ltd., Seoul, Korea) for 4 weeks. The patients in the control group (E group) received 40-mg esomeprazole alone for the same period.

Compliance was calculated by the number of remaining capsules or tablets per drug type remaining after 4 weeks. If the drug compliance exceeded $80 \%$, the data of the patient were included in outcome measurements.

\section{Outcome measurements}

The symptoms of the enrolled patients were evaluated at screening using a symptoms questionnaire, which assessed two reflux symptoms (heartburn and acid regurgitation) and four other digestive symptoms (epigastric pain, postprandial fullness, belching, and nausea). Each symptom was scored from 0 to 4 according to severity: none (no symptoms), mild (symptoms easily tolerated and did not last long), moderate (symptoms caused some discomfort but did not interfere with usual activities), severe (symptoms caused much discomfort and interfered with usual activities), or disabling (symptoms unbearable and interfered considerably with usual activities), respectively. The frequency was scored from 0 to 5: once a month or less, two to three times a month, once a week, two to three times a week, four to six times a week, or daily, respectively. The score for each symptom was calculated by multiplying its severity by its frequency. The total score for a patient was determined by the 
sum of the six symptom category scores and ranged from 0 (no symptoms) to 120 (severe symptoms) by the modified method of Fass. ${ }^{11}$ The enrolled patients were supplied with a symptoms diary, and symptom improvement was assessed weekly over the 4-week period. The primary efficacy end point was the mean decrease in the total symptom score at 4 weeks after commencing the trial medication. The secondary efficacy end points included the mean decrease in the total symptom score at 2 weeks after commencing the trial medication, the change in reflux symptoms (heartburn and regurgitation) from baseline, and the proportion of patients with at least a 50\% decrease in their symptom score compared with their baseline score.

\section{Sample size and statistical analysis}

We estimated a sample size of at least 442 to achieve a statistical power of $80 \%$ at a 5\% significance level for a two-sided test, assuming that differences between groups in the means and the standard deviations of the total symptom scores would decrease by at least 4 and 15 points over the 4-week treatment period, respectively. Anticipating a 10\% dropout rate, this study was designed to enroll 492 patients.

All analyses were performed based on an intention-totreat (ITT) or per-protocol (PP) model. Statistical analyses were performed using the two-sample t-test or paired t-test, where appropriate, for continuous data and the chi-square test for categorical data. All statistical analyses were performed using
STATA version 11.0 (StataCorp, College Station, TX, USA).

\section{RESULTS}

\section{Allocation of the patients}

This trial was conducted from November 2011 to April 2014. A total of 545 patients were screened for eligibility: 44 failed the screening, while the remaining 501 were enrolled in this study and randomized. Among the 501 patients, 240 (the ER group) were randomized to receive 40-mg esomeprazole plus 300-mg rebamipide daily, while 261 (E group) received 40-mg esomeprazole daily. Seven patients with no record of investigational product administration due to loss at follow-up were excluded from the ITT population. Subsequently, 53 patients with withdrawal, drug discontinuation because of adverse events, poor compliance, lost to follow-up, or protocol violation were excluded from the PP population. Drug discontinuation by adverse events was observed between two treatment arms (1.7\% in the ER group vs $1.2 \%$ in the E group). The severity of all adverse events was mild or moderate. No severe adverse events were reported during the study period.

As a result, 494 patients (237 in the ER group and 257 in the E group) were included in the ITT population, while the 441 patients (198 in the ER group and 243 in the E group) who completed the study were considered to be the PP population (Fig. 1).

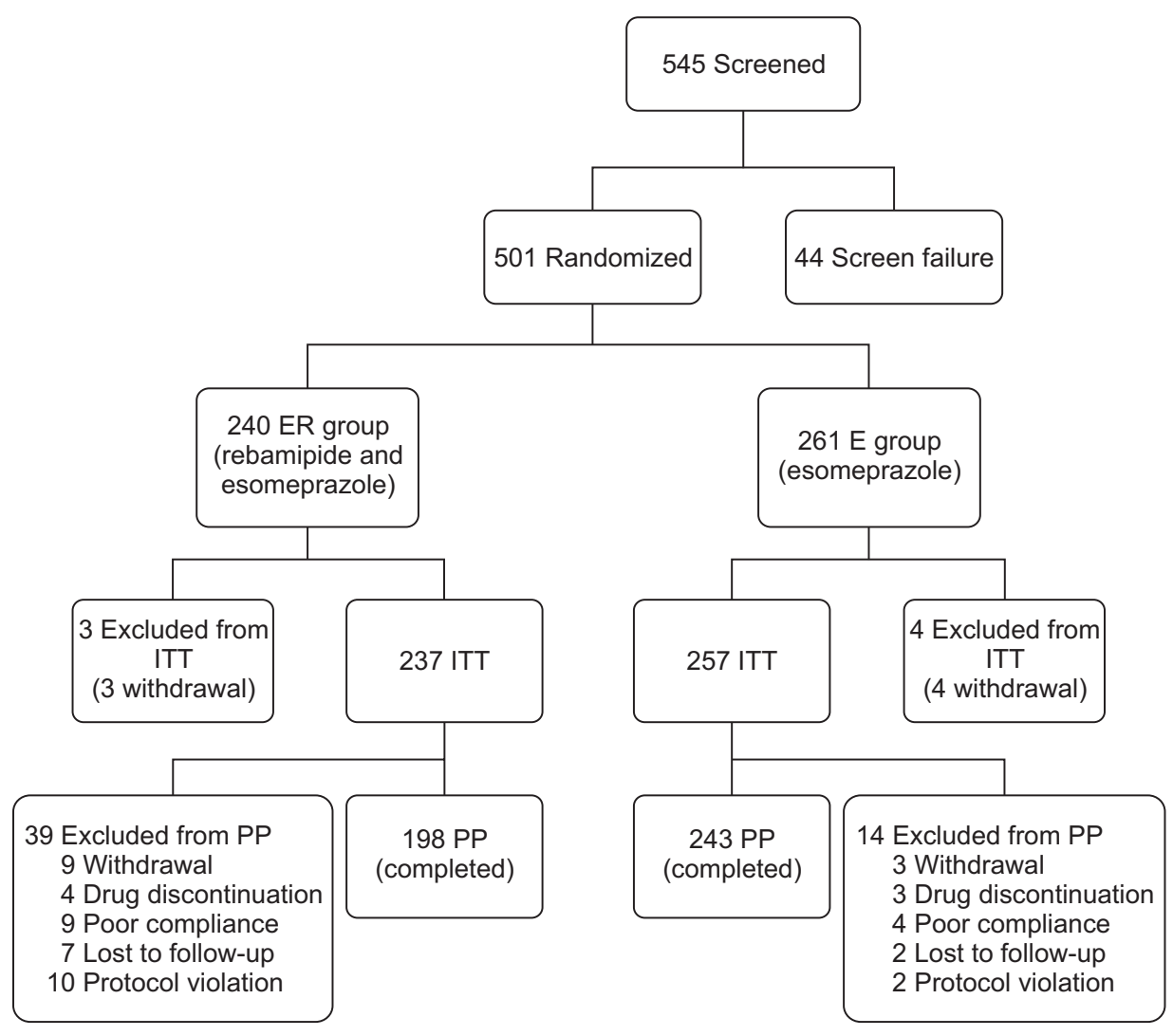

Fig. 1. Flow of patients through the study.

ITT, intention-to-treat; PP, perprotocol. 


\section{Demographics and clinical characteristics}

Table 1 demonstrates the demographic and baseline characteristics of the two groups, which showed no differences in their characteristics. The mean age of the patients was similar between the two groups. Male patients accounted for approximately $60 \%$ of the subjects in each group. The mean body weight, height, and BMI were comparable between the two treatment groups. Clinical status at baseline was also comparable in both groups of the ITT patients. Both groups showed similar severity score proportions for reflux esophagitis, presence of endoscopic gastritis, and H. pylori infection.

\section{Primary efficacy end point}

The mean total symptom score was similar in both arms at baseline and significantly decreased over the 4-week treatment period. The decrease in the total symptom score in the ER group (40-mg esomerazole plus 300-mg rebamipide daily) was significant after 4 weeks of treatment compared with that in the $\mathrm{E}$ group. The final mean decreases in the total symptom score at 4 weeks were estimated to be $-18.1 \pm 13.8$ in the ER group and $-15.1 \pm 11.9$ in the E group ( $p=0.011$ ) (Fig. 2).

\section{Secondary efficacy end point}

The mean decreases in the total symptom score after 2 weeks of treatment were $-13.4 \pm 12.9$ in the ER group and $-10.6 \pm 10.4$ in the E group ( $p=0.009$ ) (Fig. 2). Changes in reflux symptoms after 4 weeks of treatment, relative to baseline, in the ITT population were $-8.4 \pm 6.6$ in the ER group and $-6.8 \pm 5.9$ in the E group $(p=0.009)$ (Fig. 3). Two weeks after treatment, the proportions of patients who achieved reflux symptom relief of at least a 50\% decrease from baseline were $61.4 \%$ in the ER group and 52.0\% in the E group ( $\mathrm{p}=0.044)$, respectively (Table 2 ).

Table 1. Demographic and Baseline Characteristics of the Study Patients

\begin{tabular}{lccc}
\hline \multicolumn{1}{c}{ Demographic } & Rebamipide+esomeprazole $(\mathrm{n}=237)$ & Esomeprazole $(\mathrm{n}=257)$ & $\mathrm{p}$-value \\
\hline Age, yr & $49.2 \pm 13.0$ & $49.9 \pm 12.4$ & $0.544^{*}$ \\
Male sex & $146(61.6)$ & $154(59.9)$ & $0.702^{\dagger}$ \\
Weight, kg & $66.4 \pm 10.7$ & $66.6 \pm 12.0$ & $0.857^{*}$ \\
Height, cm & $165.9 \pm 8.2$ & $166.0 \pm 8.2$ & $0.950^{*}$ \\
Body mass index, $\mathrm{kg} / \mathrm{m}^{2}$ & $24.1 \pm 3.2$ & $24.1 \pm 3.4$ & $0.970^{*}$ \\
Smoking, \% & 29.0 & 30.6 & $0.799^{\dagger}$ \\
Alcohol drinking, \% & 60.5 & 48.4 & $0.078^{\dagger}$ \\
Los Angeles classification, A/B/C/D & $159 / 69 / 3 / 1$ & $181 / 70 / 3 / 0$ & $0.697^{\dagger}$ \\
Endoscopic gastritis, \% & 83.3 & 79.7 & $0.300^{\dagger}$ \\
Helicobacter pylori infection, \% & 29.2 & 23.5 & $0.178^{\dagger}$
\end{tabular}

Data are presented as mean \pm SD or number (\%).

${ }^{*}$ Two-sample t-test; ${ }^{\dagger}$ Chi-square test.

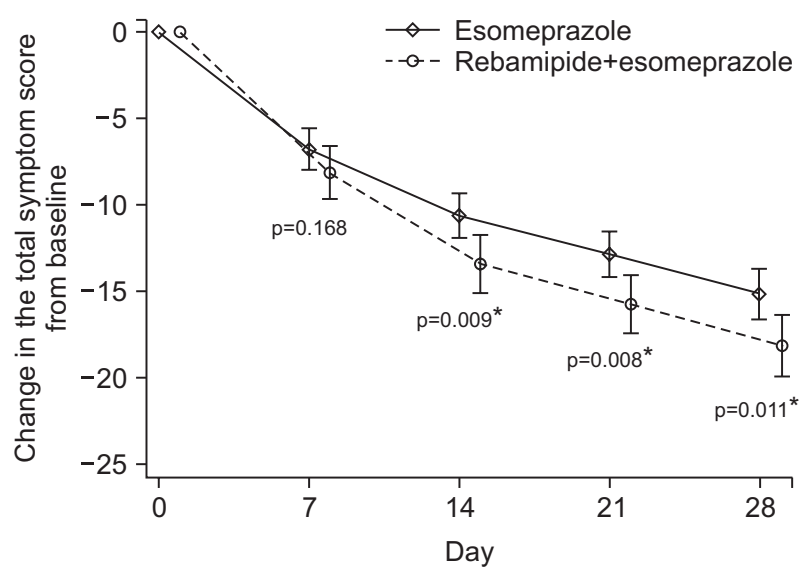

Fig. 2. Change in the total symptom score from baseline. The reduction of the total symptom score in the rebamipide+esomeprazole group (ER group) was significant at 2, 3, and 4 weeks compared with that in the esomeprazole group (E group). *Statistically significant between the reduction score in the ER group and E group.

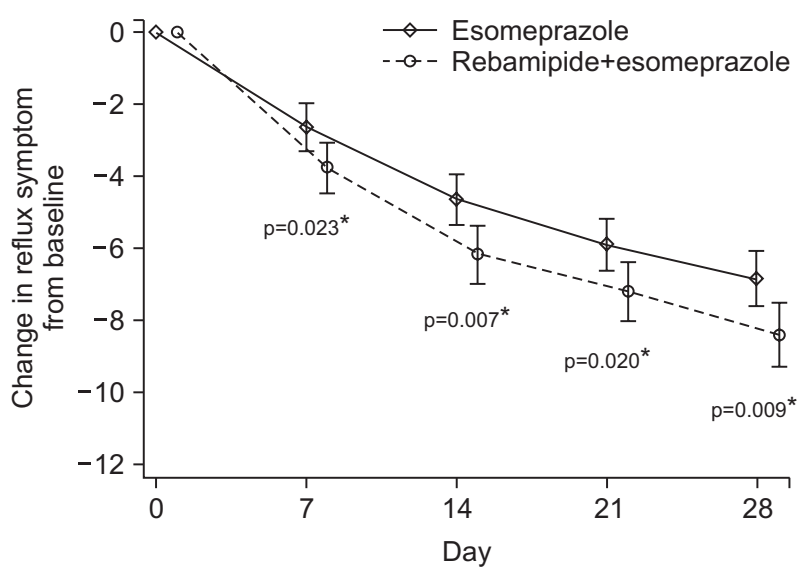

Fig. 3. Change in the reflux symptom score from baseline. The reflux symptom score in the rebamipide+esomeprazole group (ER group) was significantly decreased compared with that in the esomeprazole group (E group). *Statistically significant between the reduction score in the ER group and E group. 
Table 2. Proportion of Patients Achieving Reflux Symptom Relief of at Least a 50\% Decrease from Baseline in the Intention-to-Treat Population

\begin{tabular}{cccc}
\hline Achieving & Rebamipide+esomeprazole & Esomeprazole & p-value \\
\hline Day 7 & $83 / 222(37.4)$ & $82 / 233(35.2)$ & 0.627 \\
Day 14 & $135 / 220(61.4)$ & $120 / 231(51.9)$ & 0.044 \\
Day 21 & $162 / 216(75.0)$ & $153 / 229(66.8)$ & 0.058 \\
Day 28 & $179 / 215(83.3)$ & $180 / 229(78.6)$ & 0.213
\end{tabular}

Data are presented as number (\%).

${ }^{*}$ Chi-square test.

\section{DISCUSSION}

GERD is an inflammatory disease caused by the reflux of contents from the stomach and proximal small intestine. ${ }^{12,13}$ Hydrogen ions and pepsin contribute to the reflux injury; pepsin degrades the junctional proteins that join cells together ${ }^{14}$ and attacks vital proteins on the surface of epithelial cells. ${ }^{15}$ The increased mucosal permeability due to peptic digestion of the junctional proteins induces transportation of hydrogen ions into the deep layers of the esophageal squamous epithelium. ${ }^{14}$ Acidinduced epithelial injury promotes an inflammatory response, which is demonstrated histologically by inflammatory cells infiltrating the damaged esophageal mucosa. ${ }^{16}$

Specific cytokines and chemokines are involved in esophageal mucosal immune and inflammatory responses. In reflux esophagitis, the involvement of proinflammatory cytokines, such as IL-1 $\beta$, IL-8, and interferon- $\gamma$, was first reported by Fitzgerald et al., ${ }^{17}$ who found that the severity of reflux esophagitis was correlated with the inflammatory cell infiltration score. The expression of IL-8 mRNA measured by real-time polymerase chain reaction correlated with the endoscopic grading of reflux esophagitis according to the Los Angeles classification. ${ }^{2}$

Another study reported that the protein levels of IL-8, monocyte chemotactic protein-1, and regulated on activation normal $\mathrm{T}$ cell expressed and secreted were significantly higher in the esophageal mucosa of patients with reflux esophagitis compared with in normal subjects. ${ }^{18}$ In addition to, PPI treatment decreased IL-8 levels in the esophageal mucosa, suggesting an important role for gastric acid in the induction of esophageal inflammation. A study conducted in rats demonstrated that inflammatory cytokines and neutrophils play an important role in the pathogenesis of experimental reflux esophagitis induced by gastroduodenal content reflux. ${ }^{19}$

Oxygen-derived free radicals were also involved in the pathogenesis of esophageal mucosal injuries induced by experimental reflux esophagitis. ${ }^{20,21}$ Oxidative stress and oxidative DNA damage can develop in esophageal tissues and cells after short-term exposure to bile acids and low $\mathrm{pH}^{22,23}$ These alterations may play a role in the pathogenesis of Barrett's esophagus and tumor transformation.
A major treatment modality for acid-related diseases, such as peptic ulcers and GERD, is PPIs. The mechanism of PPI action is inhibition of acid secretion via transportation of hydrogen ions out of parietal cells by blocking the $\mathrm{H}^{+}, \mathrm{K}^{+}$-ATPase in these cells. ${ }^{24}$ As described above, inhibition of gastric acid production is important for preventing inflammation in the esophageal mucosa. Several adverse effects have been reported in subjects receiving long-term PPI therapy. ${ }^{25-28}$ Therefore, the key to PPI therapy seems to be a moderate dosage and duration of PPI administration. New drugs with other mechanisms are needed to treat GERD.

Rebamipide is a mucoprotective agent with anti-inflammatory effects due to stimulation of prostaglandin and mucous glycoprotein synthesis and inhibition of active oxygen, inflammatory cytokines and chemokines, and neutrophil activation. ${ }^{5,29}$ In an animal study, rebamipide protected against esophagitis development and reduced inflammatory mediators. ${ }^{30}$ Rebamipide was shown to reduce the adverse effects of omeprazole treatment, such as hypergastrinemia and morphological changes in the gastric mucosa of rats. ${ }^{31}$

Few clinical studies have assessed the efficacy of rebamipide in GERD. One clinical study showed that rebamipide and lansoprazole combination therapy was highly effective for preventing the recurrence of symptoms during long-term maintenance treatment for reflux esophagitis. ${ }^{8}$ Another study failed to show an advantage of rebamipide in terms of symptomatic improvement in patients with PPI-refractory nonerosive reflux disease (NERD). ${ }^{32}$ Such a discrepancy in the results originates from differences in subject populations between studies. In the study of PPI-refractory NERD, the patients did not receive ambulatory $\mathrm{pH}$ monitoring, which could have bene used to exclude patients with functional heartburn. Thus, while the histologic and antiinflammatory benefits of rebamipide are conclusive, its advantage in terms of symptomatic improvement of GERD is not clear.

Some studies have shown that inflammatory cytokines are involved in the pathogenesis of pain. ${ }^{33,34}$ IL-8 evokes hyperalgesia through a prostaglandin-independent mechanism. ${ }^{33}$ Inflammatory cytokines, such as IL-8, in the esophageal mucosa may be related to the development of reflux symptoms in patients with GERD. Rebamipide-suppressed IL-8 production may affect control of reflux symptoms in these patients.

Based on the background evidence, we designed this study to evaluate the efficacy of rebamipide and esomeprazole combination therapy during the initial therapeutic period of reflux esophagitis. In our results, rebamipide and esomeprazole combination therapy decreased symptoms more rapidly compared with esomeprazole alone in patients with reflux esophagitis. We evaluated typical reflux symptoms and other upper gastrointestinal symptoms in our patients. The changes in total symptom scores from baseline were significantly greater in the ER group than in the E group during the 4-week treatment period. Typi- 
cal reflux symptoms, including heartburn and regurgitation, also showed larger changes relative to baseline levels in the ER group. These results suggest that the combination of rebamipide and esomeprazole has a synergistic effect on symptom improvement in patients with reflux esophagitis. Further studies should investigate the mechanism of symptom improvement.

This was the first large population-based, multinational, randomized study to evaluate the efficacy of combining rebamipide with esomeprazole in patients with reflux esophagitis. However, our study had several limitations, as follows. First, it was an open-label study. Second, our study did not include patients with NERD. Third, our assessment of outcomes focused only on symptom changes in the randomized patients of the two groups. Follow-up endoscopies analyzing the healing status of esophagitis were not performed in this study.

In conclusion, combination therapy consisting of rebamipide and esomeprazole was found to be superior to esomeprazole monotherapy for symptomatic treatment of reflux esophagitis in this study. Rebamipide can be considered a relevant and effective supplementary medication for patients with reflux esophagitis. Further studies are needed to support our results and to evaluate the effects of decreasing the therapeutic dose of PPIs with rebamipide supplementation for reflux esophagitis.

\section{CONFLICTS OF INTEREST}

No potential conflict of interest relevant to this article was reported.

\section{ACKNOWLEDGEMENTS}

This research was supported by a grant from the Korean College of Helicobater and Upper Gastrointestinal Research (UMIN000005602).

\section{REFERENCES}

1. Dray A. Inflammatory mediators of pain. Br J Anaesth 1995;75: 125-131.

2. Yoshida N, Uchiyama K, Kuroda M, et al. Interleukin-8 expression in the esophageal mucosa of patients with gastroesophageal reflux disease. Scand J Gastroenterol 2004;39:816-822.

3. Labenz J, Armstrong D, Lauritsen K, et al. A randomized comparative study of esomeprazole $40 \mathrm{mg}$ versus pantoprazole $40 \mathrm{mg}$ for healing erosive oesophagitis: the EXPO study. Aliment Pharmacol Ther 2005;21:739-746.

4. Bochenek WJ, Mack ME, Fraga PD, Metz DC. Pantoprazole provides rapid and sustained symptomatic relief in patients treated for erosive oesophagitis. Aliment Pharmacol Ther 2004;20:11051114.

5. Arakawa T, Higuchi K, Fujiwara Y, et al. 15th anniversary of rebamipide: looking ahead to the new mechanisms and new ap- plications. Dig Dis Sci 2005;50 Suppl 1:S3-S11.

6. Laheij RJ, Van Rossum LG, De Boer WA, Jansen JB. Corpus gastritis in patients with endoscopic diagnosis of reflux oesophagitis and Barrett's oesophagus. Aliment Pharmacol Ther 2002;16:887891.

7. Chitapanarux T, Praisontarangkul OA, Lertprasertsuke N. An open-labeled study of rebamipide treatment in chronic gastritis patients with dyspeptic symptoms refractory to proton pump inhibitors. Dig Dis Sci 2008;53:2896-2903.

8. Yoshida N, Kamada K, Tomatsuri N, et al. Management of recurrence of symptoms of gastroesophageal reflux disease: synergistic effect of rebamipide with $15 \mathrm{mg}$ lansoprazole. Dig Dis Sci 2010;55:3393-3398.

9. Lundell LR, Dent J, Bennett JR, et al. Endoscopic assessment of oesophagitis: clinical and functional correlates and further validation of the Los Angeles classification. Gut 1999;45:172-180.

10. Tytgat GN. The Sydney System: endoscopic division. Endoscopic appearances in gastritis/duodenitis. J Gastroenterol Hepatol 1991;6:223-234.

11. Fass R, Ofman JJ, Sampliner RE, Camargo L, Wendel C, Fennerty $\mathrm{MB}$. The omeprazole test is as sensitive as $24-\mathrm{h}$ oesophageal $\mathrm{pH}$ monitoring in diagnosing gastro-oesophageal reflux disease in symptomatic patients with erosive oesophagitis. Aliment Pharmacol Ther 2000;14:389-396.

12. Gillen P, Keeling P, Byrne PJ, Healy M, O’Moore RR, Hennessy TP. Implication of duodenogastric reflux in the pathogenesis of Barrett's oesophagus. Br J Surg 1988;75:540-543.

13. Nehra D, Howell P, Williams CP, Pye JK, Beynon J. Toxic bile acids in gastro-oesophageal reflux disease: influence of gastric acidity. Gut 1999;44:598-602.

14. Tobey NA, Hosseini SS, Caymaz-Bor C, Wyatt HR, Orlando GS, Orlando RC. The role of pepsin in acid injury to esophageal epithelium. Am J Gastroenterol 2001;96:3062-3070.

15. Thangarajah H, Wong A, Chow DC, Crothers JM Jr, Forte JG. Gastric H-K-ATPase and acid-resistant surface proteins. Am J Physiol Gastrointest Liver Physiol 2002;282:G953-G961.

16. Orlando RC. Pathophysiology of gastroesophageal reflux disease. J Clin Gastroenterol 2008;42:584-588.

17. Fitzgerald RC, Onwuegbusi BA, Bajaj-Elliott M, Saeed IT, Burnham WR, Farthing MJ. Diversity in the oesophageal phenotypic response to gastro-oesophageal reflux: immunological determinants. Gut 2002;50:451-459.

18. Isomoto H, Wang A, Mizuta Y, et al. Elevated levels of chemokines in esophageal mucosa of patients with reflux esophagitis. Am J Gastroenterol 2003;98:551-556.

19. Yamaguchi T, Yoshida N, Tomatsuri N, et al. Cytokine-induced neutrophil accumulation in the pathogenesis of acute reflux esophagitis in rats. Int J Mol Med 2005;16:71-77.

20. Wetscher GJ, Hinder RA, Bagchi D, et al. Reflux esophagitis in humans is mediated by oxygen-derived free radicals. Am J Surg 1995;170:552-556.

21. Wetscher GJ, Perdikis G, Kretchmar DH, et al. Esophagitis in 
Sprague-Dawley rats is mediated by free radicals. Dig Dis Sci 1995;40:1297-1305.

22. Dvorak K, Payne CM, Chavarria M, et al. Bile acids in combination with low $\mathrm{pH}$ induce oxidative stress and oxidative DNA damage: relevance to the pathogenesis of Barrett's oesophagus. Gut 2007;56:763-771.

23. Dvorak K, Fass R, Dekel R, et al. Esophageal acid exposure at $\mathrm{pH}<$ or $=2$ is more common in Barrett's esophagus patients and is associated with oxidative stress. Dis Esophagus 2006;19:366-372.

24. Fellenius E, Berglindh T, Sachs G, et al. Substituted benzimidazoles inhibit gastric acid secretion by blocking $(\mathrm{H}++\mathrm{K}+)$ ATPase. Nature 1981;290:159-161.

25. Yokota T, Matsui H, Matsuura B, Maeyama K, Onji M. Direct effects of proton pump inhibitors on histamine release from rat enterochromaffin-like cells. Eur J Pharmacol 2003;481:233-240.

26. Haga Y, Nakatsura T, Shibata Y, et al. Human gastric carcinoid detected during long-term antiulcer therapy of $\mathrm{H} 2$ receptor antagonist and proton pump inhibitor. Dig Dis Sci 1998;43:253-257.

27. Lo WK, Chan WW. Proton pump inhibitor use and the risk of small intestinal bacterial overgrowth: a meta-analysis. Clin Gastroenterol Hepatol 2013;11:483-490.
28. Sheen E, Triadafilopoulos G. Adverse effects of long-term proton pump inhibitor therapy. Dig Dis Sci 2011;56:931-950.

29. Higuchi K, Tanigawa T, Hamaguchi M, et al. Comparison of the effects of rebamipide with those of cimetidine on chronic gastritis associated with Helicobacter pylori in Mongolian gerbils. Aliment Pharmacol Ther 2003;18 Suppl 1:1-7.

30. Katada K, Yoshida N, Isozaki Y, et al. Prevention by rebamipide of acute reflux esophagitis in rats. Dig Dis Sci 2005;50 Suppl 1:S97S103.

31. Hagiwara T, Mukaisho K, Ling ZQ, Sakano T, Sugihara H, Hattori T. Rebamipide contributes to reducing adverse effects of long-term administration of omeprazole in rats. Dig Dis Sci 2007;52:988994.

32. Adachi K, Furuta K, Miwa H, et al. A study on the efficacy of rebamipide for patients with proton pump inhibitor-refractory nonerosive reflux disease. Dig Dis Sci 2012;57:1609-1617.

33. Cunha FQ, Lorenzetti BB, Poole S, Ferreira SH. Interleukin-8 as a mediator of sympathetic pain. Br J Pharmacol 1991;104:765-767.

34. Summer GJ, Romero-Sandoval EA, Bogen O, Dina OA, Khasar SG, Levine JD. Proinflammatory cytokines mediating burn-injury pain. Pain 2008;135:98-107. 